\title{
Hydrodynamic Properties of Benthic Marine Crustacea. II. Energy Requirements for Maintaining Vertical Position
}

\author{
D. H. Spaargaren \\ Netherlands Institute for Sea Research, Postbox 59, Texel, The Netherlands
}

\begin{abstract}
In order to characterize and compare swimming abilities of crustaceans, attempts were made to assess the minimum power requirements for maintaining a constant position in a water column. The relatively slow swimming movements were compared with the flight of a helicopter; for this equations are available for calculating power during flight. A new equation is given which avoids the uncertainties introduced by using helicopter equations; the values obtained are of the same order of magnitude. The energy consumption per gram animal is very similar for the species compared. This is the more remarkable as large differences exist in their body density and drag coefficient for sinking. In large-sized species the increase in power requirement for position holding at increasing body weight is usually very small.
\end{abstract}

\section{INTRODUCTION}

In most aquatic crustaceans, specific body gravity exceeds the gravity of the surrounding medium. Hence, in absence of swimming movements they tend to sink. Many differences in swimming ability exist (for crustaceans summarized by Lockhead, 1977). Some species are forced to dwell on the bottom and can leave the substratum only for very short periods, others spend their entire life suspended in the water column. These differences are not easily related to the density or the drag coefficient for sinking (Spaargaren, 1980a).

When body density exceeds medium density, energy is required to maintain suspension. The amount of energy needed per unit time (the power requirement) probably varies considerably. Of course, velocity of locomotion significantly affects frictional resistance. Further, the weight (underwater weight) is of importance. If we wish to assess and compare power requirements of crustaceans, these factors must be quantified.

Denton (1961) already noted the difficulties which must be expected in attempts to assess power requirements for swimming. A certain minimum power is required in order to keep the animal suspended. This minimum power depends on the animal's hydrody- namic properties and on the medium (Nachtigall, 1977; Wu, 1977). Estimates of minimum power requirement should make it possible to quantify swimming abilities and to explain variations in locomotory behaviour.

This paper compares the usefulness of a few equations which can be used for assessing the minimal power required to remain suspended.

\section{MATERIAL AND METHODS}

Power requirements for swimming of the following crustaceans were considered: Palaemon serratus (Pennant), Lysmata seticaudata (Risso), Sicyonia carinata (Olivi), Pontophilus spinosus (Leach), Crangon crangon (L.), Praunus flexuosus (Müller), Idothea balthica (Pallas), Homarus vulgaris (L.) and Maia squinado (Herbst). Data regarding body density and drag coefficient for sinking of these species were obtained from Spaargaren (1980).

Three equations were used; two of these are commonly used in aerodynamic work (e. g. Gessow and Myers [Chapter 4], 1952; Vay, 1967); the third is derived below. 


\section{RESULTS AND DISCUSSION}

It seems attractive to compare the locomotion dynamics of crustaceans with those of helicopters. In both cases the horizontal velocities are relatively small and both can maintain a constant vertical position in the absence of horizontal movements.

For a helicopter in standing-flight the power induced by the rotor is given by:

$$
P_{o}=\sqrt{\frac{(m g)^{3}}{2 \pi r^{2} \varrho}}
$$

$\left(P_{o}\right.$ : induced power $;$ r: radius of the rotor; $m$ : mass; $g$ : gravitational constant; $\varrho$ : density of air). In forward flight, the power requirement decreases in relation to the horizontal speed $(v)$ according to:

$$
p_{v}=\frac{(m g)^{2}}{2 \pi r^{2} \varrho v}
$$

(in using cgs-units both equations result in power values expressed in erg s $\mathrm{s}^{-1} ; 1 \mathrm{erg} \mathrm{s}^{-1}$ equals $0.1 \mu \mathrm{W}$ ).

Can those equations be applied to aquatic animals? Here the dimensions and velocities are smaller, the medium density larger. Applicability seems justified only on condition that the Reynolds number, $R e=x \cdot v \cdot S_{\mathrm{m}} / \eta=x \cdot v / v(x$ : dimension; $v$ : kinematic viscosity; $v$ : velocity; $S_{m}$ : medium density; $\eta$ : static viscosity) remains approximately the same. An exact comparison between the Reynolds numbers of a helicopter and those of the animals considered here is not possible. In building helicopters one attempts to keep the Reynolds number as low as possible. From previous experiments (Spaargaren, 1980a) it appeared that, in sinking movements, the drag force remains proportional to the sinking rate, indicating a nonturbulent flow passing the animal as expected with low values for the Reynolds number. In these animals the low Reynolds numbers are related to (1) their small dimensions, (2) low locomotory velocities and (3) high medium viscosity. For a helicopter the very low density of air may partly compensate for the larger dimensions, the higher velocities and the lower viscosity, but the helicopter $R e$ will remain much higher than that for a crustacean.

These differences introduce uncertainties in the application of Equations (1) and (2) to aquatic animals. A further disadvantage is that the surface area of the locomotory organs (the factor $\pi r^{2}$ in Equation (1) and Equation (2) in crustaceans) cannot be given very accurately.

Another equation may be more appropriate for the crustaceans considered. In the absence of a locomotory force the crustacean sinks, practically instantaneously, with a constant speed. The halftime for reaching equi- librium velocity is $t_{1 / 2}=0.693 \mathrm{~m} / \mathrm{k}$ ( $\mathrm{m}$ : fresh weight; $k$ : drag coefficient); even in large crustaceans like Palaemon serratus with $m=1 \mathrm{~g}$ and $k=19.61 \mathrm{gs}^{-1}, t_{1 / 2}$ is very short: $0.035 \mathrm{~s}$. During sinking an amount of potential energy is dissipated at a constant rate given by the product of underwater weight $\left(G_{s}=m g\right.$ $\left(1-\frac{S_{m}}{S_{a}}\right)$ and velocity:

$$
P=m g\left(1-\frac{S_{m}}{S_{a}}\right) \cdot v_{\infty}
$$

Terminal velocity, $V_{\infty}$, is given by: $m g\left(1-S_{m p} / S_{a}\right) / \mathrm{k}$. Hence,

$$
P=G_{s}^{2} / k \cdot v_{\infty}^{2}
$$

To regain the potential energy, the crustacean must at least spend the same amount of energy as was lost during sinking. However, during ascent the upward velocity also induces a friction force; to overcome this friction force an additional amount of energy is required. This loss will tend to zero in keeping a constant position in the vertical when the vertical speed is reduced; in that case the power induced by the locomotory force will be given by Equation (3).

Calculation of power requirements for maintenance of a constant, vertical position with the three equations available for a shrimp (fresh weight: $1 \mathrm{~g}$; drag coefficient $k$ : $20 \mathrm{~g} \mathrm{~s}^{-1}$; pleopod surface area: $2.26 \mathrm{~cm}^{2}$; estimated radius: $0.6 \mathrm{~cm}$ ) yields according to Equation (1): $P_{o}=64 \mu \mathrm{W}$ att. According to Equation (2) $P_{v}$ depends on horizontal velocity $\left(v=1-6 \mathrm{~cm} \mathrm{~s}^{-1}\right): P_{1}=415$, $\mathrm{P}_{2}=208, \mathrm{P}_{3}=139, \mathrm{P}_{4}=104, \mathrm{P}_{5}=83, \mathrm{P}_{6}=69 \mu \mathrm{W}$ att. Equation (3) yields: $\mathrm{P}=96 \mu \mathrm{W}$ att. The difference between $P_{o}$ and $P$ could very well have been caused by the inaccuracy in estimating pleopod surface area, necessary for calculating $P_{\circ}$. Equation (2) yields at the various velocities $\left(1-6 \mathrm{~cm} \mathrm{~s}^{-1}\right)$ higher as well as lower values as those found using Equation (3). Equation (2) is usually considered invalid at velocities resulting in $P$-values exceeding those obtained with Equation (1). Here, this is the case up to velocities of about $6 \mathrm{~cm} \mathrm{~s}^{-1}$ The order of magnitude for $P$ using the three different equations is about the same; as in Equation (3) the estimate of the surface area of the locomotory organ is lacking, this equation will be more appropriate for the animals considered here.

Maintenance of a vertical position without horizontal movement is energetically unfavourable. While the minimal power requirement may be lower, there are two reasons to assume that the difference between the power requirement calculated according to Equation (3) and the minimum power requirement will only be small: (i). Normal shrimp swimming speeds are low and usually the animals only show catadromous activity (e. g. Spaargaren, in prep.); this also implies that their velocity, with regard to the surrounding medium, is low and probably does not contribute very much to 


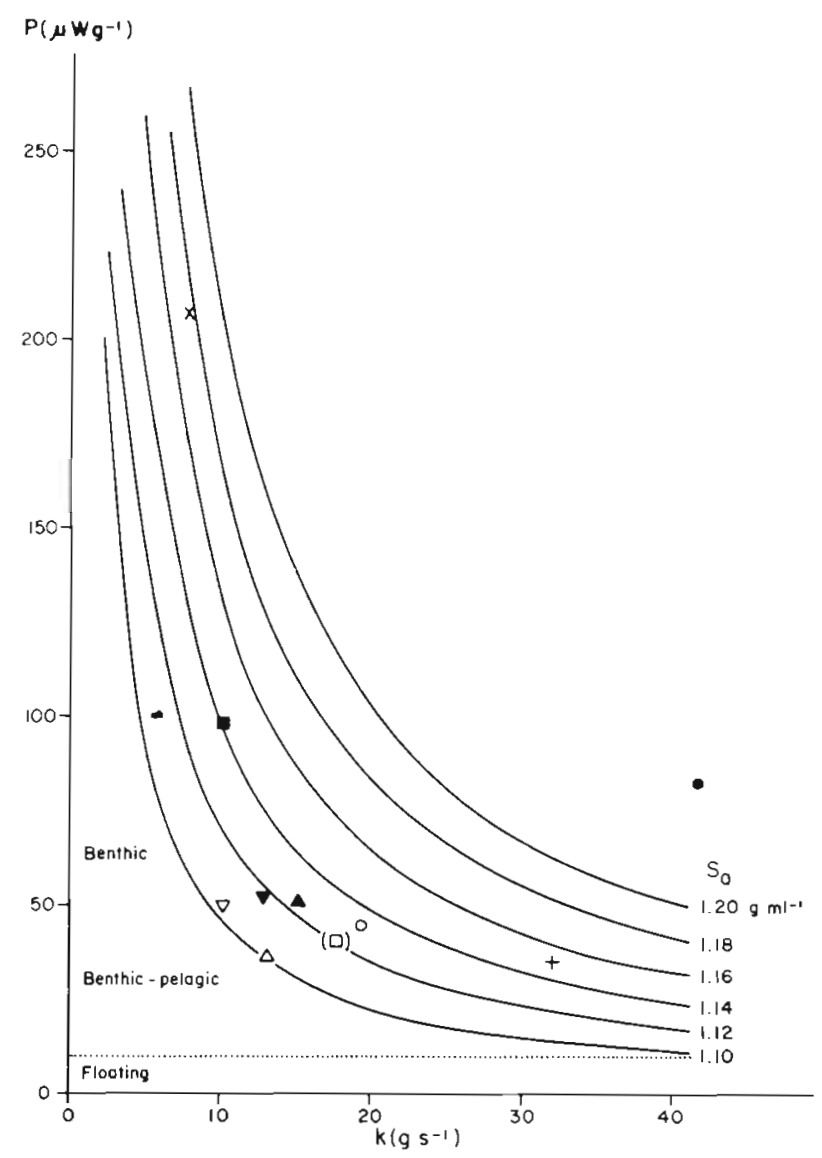

Fig. 1. Power required for maintaining a crustacean of $1 \mathrm{~g}$ (fresh weight) at a fixed vertical position in the water column, expressed as function of drag coefficient for sinking $(k)$ and body density $\left(S_{\mathrm{a}}\right)$ : Palaemon serratus (o); Palaemon adspersus $(\bullet)$; Lysmata seticaudata (ه); Sicyonia carinata (\$); Crangon crangon ( $\nabla)$; Pontophilus spinosus ( $\nabla$ ); Homarus vulgaris (ㅁ); Idothea balthica (-); Praunus flexuosus (+); Maia squinado ( $\times$ ). Temperature $20^{\circ} \mathrm{C}$; salinity ca $35 \% \mathrm{~S}$ an upward lift. This is also indicated by the fact that from Equation (2), even at a velocity of $6 \mathrm{~cm} \mathrm{~s}^{-1}$, the value calculated for $P$ is still higher than $P_{o}$ (ii) the crustaceans examined do not show much streamline in their body shape. This implies that the advantage given by a horizontal velocity will soon be lost in the increase of so-called parastic powers (the energy consumption necessary to overcome the resistance against horizontal movements).

Other factors might render the real energy demand of the animal considerably higher than the values for induced power: if we assume metabolic energy to be converted to locomotory energy with an efficiency of about $20 \%$, then the energy consumption of the animal will be 5 times larger than the values following from the above equation.

If we compare the values for induced power (from above calculations about $100 \mu \mathrm{Watt}$ ) with the maximal power dissipation ( $1 \mathrm{mWatt}$ ), which can roughly be estimated for an individual of $1 \mathrm{~g}$, then it is evident that, with a conversion efficiency of $20 \%$, swimming may require a large part $(50 \%)$ of the total energy budget.

Table 1 shows values for $P$ which, using Equation (3) and data on animal density and drag coefficients for sinking, can be obtained for various species. Values for individuals of $1 \mathrm{~g}$ are indicated in Figure 1, showing the relation between power requirement, drag coefficient and density. With low values for the drag coefficient the power requirement is strongly dependent on the density, while with high drag-coefficient values the power requirements are much lower and specific gravity has little influence. This phenomenon is also demonstrated in the distribution of water droplets in air: small droplets may float in air because their fric-

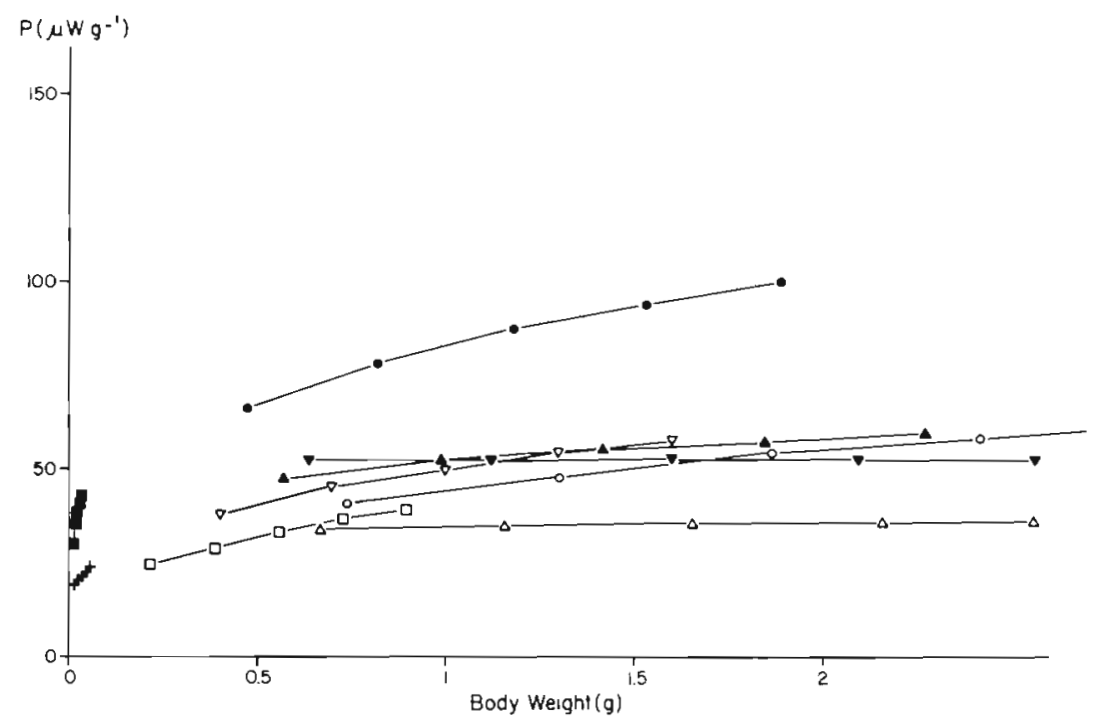

Fig. 2. Power required per $g$ fresh weight for maintaining a fixed vertical position in the water column, expressed as a function of body weight. Symbols: see legend to Figure 1 
Table 1. Specific gravity $\left(S_{a}\right.$ in $\left.\mathrm{g} \mathrm{m}^{-1}\right)$, drag coefficient for sinking $\left(k\right.$ in $\left.\mathrm{g} \mathrm{s}^{-1}\right)$ and power requirement for maintaining a fixed vertical position ( $P$ in erg s $\mathrm{s}^{-1} \mathrm{~g}^{-1}$ ) in the crustaceans listed for animals of $1 \mathrm{~g}$ (fresh weight). The values for $a$ and $b$ give the weight influence on respectively drag coefficient and power requirement: for an animal of $m$ gram drag coefficient and power requirement follow from respectively: $k_{m}=\mathrm{km}^{\mathrm{a}}$ and $P_{m}=P_{m}^{b}$

\begin{tabular}{|lccccc|}
\hline \multicolumn{1}{|c}{ Species } & $S_{\theta}$ & $k$ & $a$ & $p$ & $b$ \\
\hline Palaemon serratus & 1.1318 & 19.61 & 0.689 & 443 & 0.311 \\
Palaemon adspersus & 1.2644 & 41.92 & 0.708 & 828 & 0.292 \\
Lysmata seticaudata & 1.0981 & 12.48 & 0.968 & 348 & 0.032 \\
Sicyonia carinata & 1.1226 & 14.19 & 0.841 & 520 & 0.159 \\
Crangon crangon & 1.1044 & 10.17 & 0.699 & 498 & 0.301 \\
Pontophilus spinosus & 1.1148 & 12.26 & 0.988 & 517 & 0.002 \\
Idothea balthica & 1.1460 & 11.08 & 0.756 & 980 & 0.244 \\
Homanus vulgaris & 1.1170 & 16.24 & 0.650 & 408 & 0.350 \\
Praunus flexuosus & 1.1474 & 31.72 & 0.863 & 349 & 0.137 \\
Maia squinado & 1.1812 & 8.16 & 1.087 & 2081 & -0.087 \\
\hline
\end{tabular}

tional resistance with the medium is high, despite the fact that the density of water is much higher than that of air. It appears that, despite large differences in density and drag coefficients between the various species considered, the power requirements do not show large interspecific differences. Only the spider crab Maia squinado shows a very high value and this is also a species which is completely unable to swim. The lowest values are found for Praunus flexuosus and Lysmata seticaudata; both species can swim very well. The other species show intermediate values.

Figure 2 shows the power requirement for maintaining a fixed position in the water column in relation to animal weight. Generally the values found for different species are very similar. With increasing weight the power demand (per $g$ animal) increases; in some species, especially the larger ones, the increase is only small or practically absent. Low power requirements for swimming in juveniles (not shown in the graph, which only refers to adult individuals for which density and drag coefficient values were determined) is obviously important for the distribution of a species.

Acknowledgement. I am much indebted to ing. H. J. M. Zijm (Institute of Naval Aeronautics, Den Helder) for his interest and valuable advice.

\section{LITERATURE CITED}

Denton, E. J. (1961). The buoyance of fishes and cephalopods. Prog. Biophys. 11: 177-235

Gessow, A. and G. C. Myers (1952). Aerodynamics of the helicopter, MacMillan, New York

Lockhead, J. H. (1977). Unsolved problems of interest in the locomotion of crustacea. In: Pedley, T. J. (ed.) Scale effects in locomotion. Academic Press, London

Nachtigall, W. (1977). Swimming mechanics and energetics of locomotion of various sized water beetles (Dytiscidae, body length 2-35 mm). In: Pedley, T. J. (ed.) Scale effects in locomotion. Academic Press, London

Spaargaren, D. H. (1980). Hydrodynamic properties of benthic marine crustacea. I. Specific gravity and drag coefficients. Mar. Ecol. Prog. Ser. 1: 351-359

Spaargaren, D. H. (in prep.). The significance of seawater viscosity for the tidal transport of common shrimps, Crangon crangon (L.)

Vay, J. (1967). The helicopter and how it flies, Pittman, London

Wu, T. Y. (1977). Introduction to the scaling of aquatic animal locomotion. In: Pedley, T. J. (ed.) Scale effects in locomotion. Academic Press, London 